\title{
Drug Mimicry: Promiscuous Receptors PXR and AhR, and Microbial Metabolite Interactions in the Intestine
}

Zdeněk Dvořák, ${ }^{1, \star}$ Harry Sokol, ${ }^{2,3,4}$ and Sridhar Mani, ${ }^{5,6,7, *}$

Significant attrition limits drug discovery. The available chemical entities present with drug-like features contribute to this limitation. Using specific examples of promiscuous receptor-ligand interactions, a case is made for expanding the chemical space for drug-like molecules. These ligand-receptor interactions are poor candidates for the drug discovery process. However, provided herein are specific examples of ligand-receptor or transcription-factor interactions, namely, the pregnane $\mathrm{X}$ receptor (PXR) and the aryl hydrocarbon receptor (AhR), and its interactions with microbial metabolites. Discrete examples of microbial metabolite mimicry are shown to yield more potent and non-toxic therapeutic leads for pathophysiological conditions regulated by PXR and AhR. These examples underscore the opinion that microbial metabolite mimicry of promiscuous ligand-receptor interactions is warranted, and will likely expand the existing chemical space of drugs.

\section{Expanding the Chemical Space of Drugs via Microbial Metabolite Mimicry of} Promiscuous Metabolite-Receptor Interactions

Promiscuous receptor-ligand interactions, while on the one hand are problematic for necessary science investigations into receptor biology, maybe a treasure trove for investigating the bounds of chemical space available for that receptor [1]. If the receptor per se, influences a biological phenomenon, then there is theoretical potential to develop more potent small molecules mimicking that chemical space $[2,3]$. To mine this concept, we start with orphan or adopted orphan nuclear receptors, as it is well established that these sets of receptors, and related ligand-activated transcription factors, have significant ligand promiscuity [4].

Orphan Nuclear Receptors and Transcription Factors as Attractive Targets for Host Modulation of Physiology and Pathophysiology

The absence of a well-defined endogenous ligand defines 'orphan nuclear receptors'. Once the endogenous ligand is discovered, the terms, 'deorphanization' or 'adopted orphan receptors' are used. The most studied subgroups of the orphan nuclear receptors comprise xenobiotic receptors, and metabolic and energy sensors. Unlike nuclear hormone receptors, orphan nuclear receptors have a large ligand-binding domain, and they are promiscuous in binding a wide array of structurally unrelated compounds. Also, ligands of orphan nuclear receptors usually have a lower affinity and potency than nuclear hormone receptors. Therefore, orphan nuclear receptors (salient receptors are elaborated on later) are sometimes referred to as high-capacity and lowspecificity (affinity) receptors.

The discovery of the first xenosensor, the AhR, a ligand activated transcription factor, dates back to the early 1970s. The AhR is activated by several xenobiotics, including environmental

\section{Highlights}

The lack of chemical diversity in drug-like molecules is one important reason for drug attrition.

Microbial metabolite mimicry of promiscuous ligand-receptor interactions is warranted, and will likely expand the existing chemical space of drugs.

Prototypical host receptors that exhibit weak ligand interactions include xenobiotic nuclear receptors such as PXR and AhR.

Using PXR and AhR as exemplar xenobiotic receptors, discrete examples of microbial metabolite mimicry, are shown to yield more potent and nontoxic therapeutic leads, for pathophysiological conditions regulated by these receptors.

${ }^{1}$ Departments of Cell Biology and Genetics, Palacký University, Olomouc 78371, Czech Republic

${ }^{2}$ Sorbonne Université, Inserm, Centre de Recherche Saint-Antoine, CRSA, AP-HP, Hôpital Saint Antoine, Service de Gastroenterologie, F-75012 Paris, France

${ }^{3}$ INRA, UMR 1319 Micalis and AgroParisTech, 78352 Jouy-en-Josas, France

${ }^{4}$ Paris Centre for Microbiome Medicine FHU, Paris, France

${ }^{5}$ Department of Molecular

Pharmacology, Albert Einstein College of Medicine, Bronx, NY 10461, USA

${ }^{6}$ Department of Genetics, Albert Einstein College of Medicine, Bronx, NY 10461, USA

${ }^{7}$ Department of Medicine, Albert Einstein College of Medicine, Bronx, NY 10461, USA 
pollutants, plant polyphenolics, alkaloids, synthetic compounds, and drugs. The examples of endogenous AhR ligands are eicosanoids, indirubin, bilirubin, or 6-formylindolo[3,2-b]carbazole (FICZ) [5]. Whereas the AhR was considered for a long time as a transcriptional mediator of xenoprotective and drug-metabolizing genes, there is a substantial body of evidence for the involvement of the AhR in many physiological processes, such as hematopoiesis, restorative neurogenesis, organ development, embryogenesis, and immunity [6]. Moreover, the AhR is a pivotal actor in the pathogenesis of cancer [7], intestinal inflammation [8], hepatic steatosis [9], and atopic dermatitis [10]. Given the broad and essential roles of the AhR in human physiology and pathophysiology, the attempts for therapeutic targeting of the AhR have emerged. Multiple reports proposed the AhR signaling pathway as a target for anticancer therapy, including a strategy of repurposing existing drugs [7,11]. Clinically approved AhR active drugs lansoprazole, omeprazole, raloxifene, flutamide, sulindac, tranilast, leflunomide, nimodipine, or mexeletine were suggested as off-target chemotherapeutics for the treatment of breast cancers [12]. Off targeting the AhR with tranilast was used in the treatment of atopic dermatitis [13]. Topical application of coal tar induced skin barrier repair in atopic dermatitis via the AhR pathway [14]. A topical cream containing tapinarof, a bacterial stilbenoid, and an AhR agonist, was examined, as an agent for atopic dermatitis [10] and plaque psoriasis [15]. The roles of the AhR in the pathogenesis of inflammatory bowel diseases are widely known [16], and the insufficient or inappropriate activation of the AhR is associated with inflammatory conditions [8]. Whereas the activation of the intestinal AhR by various xenobiotics was reported to trigger and to attenuate colitis, therapeutic targeting of the intestinal AhR was not applied to date. Interestingly, very few AhR antagonists described, and more recently, natural vitamins, folate, and B12, seem to function as endogenous AhR antagonists [17].

The PXR was initially described as an orphan nuclear receptor, activated by natural and synthetic steroids. In this context, it is important to recognize that the mouse and the human receptors respond to distinct ligands (e.g., the mouse receptor is activated by pregnenolone carbonitrile and the human receptor is activated by rifampicin), and therefore, displays species-specificity. In this context, with respect to intestinal microbial metabolites, indolyl-3-propionate (IPA) is a mouse PXR ligand, but not an efficient human PXR ligand. However, combinatorial indole metabolite mixtures can activate the human PXR receptor. These metabolites are produced in both rodent and human intestines. Thus, there is specificity in terms of the types of ligands found, as microbial metabolites and their ligand activation of PXR. For translation to human conditions, human PXR ligands also necessitate the use of humanized mice and these mice have been created by several groups and companies (e.g., Taconic). The PXR is activated by xenobiotics that induce drug-metabolizing enzymes, causing pharmacokinetic drug interactions, which is undesired [18]. Xenobiotic ligands of the PXR are structurally unrelated compounds, including drugs (e.g., rifampicin), natural compounds (e.g., hyperforin and solomonsterols) and environmental pollutants (e.g., phthalates and bisphenols) [19,20]. Currently, the PXR is 'deorphanized' and endogenous ligands such as bile acids, estrogens, or vitamin $\mathrm{K} 2$, were identified. Besides the central role in the regulation of xenobiotic-metabolizing enzymes, the PXR controls the intermediary metabolism of lipids, glucose, and bile acids, and it is involved in the etiology of various diseases such as diabetes, metabolic syndrome, cardiovascular pathologies, inflammatory bowel disease, acute kidney injury, neurological pathologies, and cancer [20]. Hence, the therapeutic targeting of the PXR with both agonists and antagonists is of particular interest [19,20].

The constitutive androstane receptor (CAR) is a 'sister' receptor of the PXR [21], because they share and overlap ligands and activators, transcriptional partners, specific response elements in DNA, a set of target genes, and in general, biological functions [22]. In contrast to other nuclear receptors, the CAR constitutively activates gene transcription. Initially, it was observed that the *Correspondence:
zdenek.dvorak@upol.cz (Z. Dvořák) and
sridhar.mani@einsteinmed.org (S. Mani) 
steroids androstanol and androstenol inhibit the constitutive activity of the CAR (inverse agonist effects) by a mechanism involving the promotion of coactivator release from the ligand-binding domain. Therefore, an unanticipated steroidal signaling pathway that functions in a manner opposite to that of the conventional nuclear receptor pathways was defined [21]. Targeting the CAR in the therapy of metabolic diseases, cancer, diabetes, inflammatory, and liver diseases, were proposed [23].

The liver $X$ receptor $(L X R)$ exists in two forms, (i.e., $L X R \alpha$ and $L X R \beta)$, which have tissue-specific expression. The endogenous ligands of the LXRs are oxysterols, and intermediates of the mevalonate metabolic pathway. An example of the xenobiotic activator is synthetic compound GW3965. Physiological roles for the LXRs are the regulation of glucose, fatty acids, and cholesterol metabolism. Therefore, the LXRs are promising targets for the therapy of obesity, diabetes, and atherosclerosis [24]. Therapeutic targeting of the LXRs, using dual or LXR $\alpha / L X R \beta$-selective ligands, is also considered in cancer [25], and inflammatory and neurodegenerative diseases [26]. Oltipraz, an antagonist of the LXRa, reduced the liver fat content in patients with nonalcoholic fatty liver disease (NAFLD) in phase II clinical trial [27]. The LXRß-selective agonist BMS852927, increased reverse cholesterol transport pathways, but adverse effects, such as elevated LDL cholesterol and triglycerides, were reported [28]. An improvement in barrier differentiation and lipids, was observed in patients with mild to moderate atopic dermatitis, topically administered with LXRß-selective ligand VTP-38543 [29].

The farnesoid X receptor (FXR; NR1H4) was named after its first identified ligand farnesol. Endogenous ligands of the FXR are bile acids; hence the FXR is sometimes referred to as bile acid receptor. The physiological function of the FXR is the regulation of the metabolism of bile acids, fatty acids, triglycerides, and glucose. Consistently, FXR is an attractive target for the treatment of metabolic and hepatic diseases [24,30]. Several clinical trials were carried out with highly potent FXR synthetic agonist obeticholic acid, including those against type II diabetes and NAFLD [31], non-cirrhotic nonalcoholic steatohepatitis [32], primary sclerosing cholangitis [33], and primary biliary cholangitis [34]. For the latter, FDA-approved drug Ocaliva $₫$ is available. The examples of other FXR agonists currently under clinical investigations are Cilofexor (Gilead Sciences), and PX-102 (Phenex Pharmaceuticals).

The peroxisome proliferator-activated receptors (PPARs) regulate glucose and lipid homeostasis, inflammation, proliferation, and differentiation [35]. Fatty acids activate the PPARs and their derivatives, the examples of endogenous ligands for PPAR a and PPARy are oleoylethanolamide and 15-deoxy-prostaglandin J2, respectively. Xenobiotic ligands for the PPARs are various endocrine-disrupting chemicals that cause obesogenic effects. The therapeutic target for hypolipidemic drugs of fibrates class is PPARa, whereas antidiabetes type-Il thiazolidinediones target PPARy. Targeting PPARs in cancer therapy [35] and diabetic microvascular damage [36] was proposed.

\section{Microbial Metabolites as Modulators of AhR, PXR, FXR, LXRs, and PPARs Receptors}

Human gut microbiota produces a broad spectrum of metabolic products that interact with the host organism, and mediate beneficial health effects and pathogenicity. The microbial metabolites are structurally diverse compounds, including short and medium-chain fatty acids, polysaccharides, polyamines, formyl-peptides, bile acids, tryptophan metabolites, acylhomoserine lactones, gaseous substances (hydrogen sulfide, methane), toxins, and others [37]. At cellular levels, the gut microbial metabolites interact mostly with G protein-coupled receptors and nuclear receptors, through which they mediate their biological effects both onsite and distantly. 
The following examples illustrate how multiple microbial metabolites can serve as weak but promiscuous ligands, for a given nuclear receptor, and across ligand-activated transcription factor systems. In this context only PXR and AhR are highlighted, as the other receptors mentioned, LXR, FXR, and PPAR, are not, per se, promiscuous receptors. Metabolites of tryptophan, produced by human intestinal microbiota, were extensively studied as ligands of the AhR and mediators of intestinal health and disease. To date, numerous tryptophan catabolites were identified as low-affinity AhR ligands (agonist and antagonist), including indole, skatole, tryptamine, and a series of indolyl-3-(lactate, pyruvate, acrylate, propionate, acetate, aldehyde, acetamide, ethanol) ligands [38,39]. Ligand-dependent transcriptional activation of the AhR, was synergistically enhanced by short-chain fatty acids (SCFAs) [40]. Other examples of intestinal-microbiota derived AhR ligands comprise 2,8-dihydroxy-quinoline, urolithin A (formed from ellagitannins), and 1,4-dihydroxy-2-naphthoic acid [41].

The most studied microbial PXR ligands were the indole products of tryptophan catabolism, including skatole [42], indolyl-3-acetate (IAA), and IPA; the latter being reported to diminish intestinal inflammation through PXR pathway [43]. Intestinal proinflammatory responses in the early stages of experimental necrotizing enterocolitis were attenuated by lithocholic acid, through targeting the PXR [44]. Indirect effects of various xenobiotics such as statins or green tea polyphenols through PXR, were shown to result in dysregulation of intestinal SCFAs and bile acid composition. Whereas the involvement of CAR in the intestinal mucosal response to injury was demonstrated, direct proof of CAR targeting with microbial metabolites was not provided [45].

The antioxidant tempol displayed antiobesity activity, by reducing intestinal genus Lactobacillus, leading to the accumulation of tauro- $\beta$-muricholic acid, which is the FXR antagonist [46]. Similarly, theabrownin-suppressed gut microbes, with bile-salt hydrolase activity, resulted in increased levels of taurochendoxycholic, tauroursodeoxycholic, glycochenodeoxycholic, and glycoursodeoxycholic acids, exhibiting hypolipidemic effects through inhibition of the intestinal FXR [47]. Hypoglycemic activity of metformin was partly explained by shaping gut microbiota when Bacteroides fragilis diminished, and the levels of glycoursodeoxycholic acid, an FXR antagonist, increased [48]. Recently, a new class of bile acids with FXR agonist activity, produced by human gut microbiota, was identified. These acids are conjugated with atypical amino acids, yielding novel phenylalanocholic, tyrosocholic, and leucocholic acids [49]. Overall, gut bacterial bile acids are the agonist and antagonists of the FXR, that display their biological effects partly through this receptor; however, the activation of the PXR cannot be excluded. Interestingly, there are no available data about the interactions between $L X R$ and gut microbial metabolites.

Several studies reported the activation of the intestinal PPARy by SCFAs, mainly by propionate and butyrate [50]. Depletion of butyrate-producing microbes by antibiotics reduced epithelial signaling through PPARy [51]. Probiotic bacteria producing conjugated linoleic acids locally in the gut, were shown to suppress colitis through targeting PPARY [52].

\section{Indole and Its Microbial Metabolites as Promiscuous Ligands, for a Given Receptor and across Receptor Systems}

L-tryptophan catabolism, specifically via microbes in the intestine, is essential for host-microbe symbiosis [53,54] (Figure 1). Indole per se, has been extensively studied concerning AhR. Indole is a very weak PXR ligand [43]. However, indoles ( 1 mM) can also inhibit voltage-gated K (+) channels leading to acute stimulation of GLP-1 secretion from intestinal $L$ cells; however, a more chronic effect of indoles reduces GLP-1 via inhibition of NADH dehydrogenases, and a slow loss of ATP [55]. Indole also has effects on bacteria, such that it causes spatial segregation of bacteria in the gut that resist invaders, but helps recruit beneficial organisms [56]. Additionally, 


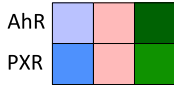<smiles>c1ccc2[nH]ccc2c1</smiles>

Indole

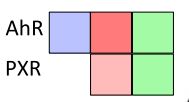<smiles>O=Cc1c[nH]c2ccccc12</smiles>

Indole-3-aldehyde

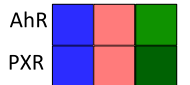<smiles>NC(=O)Cc1c[nH]c2ccccc12</smiles>

Indole-3-acetamide

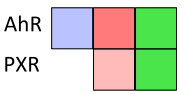<smiles>OCCc1c[nH]c2ccccc12</smiles>

Indole-3-ethanol

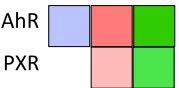<smiles>NCCc1c[nH]c2ccccc12</smiles>

Tryptamine

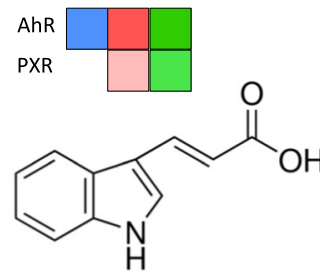

Indole-3-acrylate

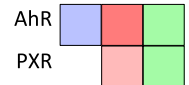<smiles>O=C(O)Cc1c[nH]c2ccccc12</smiles>

Indole-3-acetate

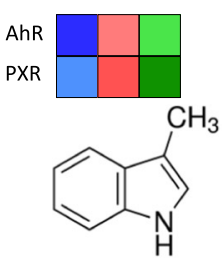

Skatole<smiles>CC1(c2ccccc2)CC2(CC2)CC1Cc1c[nH]c2ccccc12</smiles>

Indole-3-pyruvate<smiles>O=C(O)CCc1c[nH]c2ccccc12</smiles>

Indole-3-propionate<smiles>O=C(O)C(O)Cc1c[nH]c2ccccc12</smiles>

Indole-3-lactate

Figure 1. Tryptophan Intestinal Microbial Catabolites as Ligands and Agonists of Xenosensors, AhR and PXR. A chart summarizes molecular effects of known human intestinal microbial metabolites of tryptophan at AhR and PXR receptors. Heat-map shows a semi-quantitative profile of interactions: (i) Affinity, is a measure of compound binding at the receptor ligand binding domain (dissociation constant $\approx K_{D}$ ); (ii) Potency, refers to the concentration of the compound to produce $50 \%$ of maximal effect (half-maximal effective concentration $\approx \mathrm{EC}_{50}$ ); (iii) Efficacy, is the maximum response that can be reached by the compound. Abbreviations: PXR, pregnane X receptor; AhR, aryl hydrocarbon receptor

indole can attenuate the IFN1 pathway, and limit graft-versus-host disease in mice that have undergone allogeneic bone marrow transplantation [57]. IPA is a mouse PXR agonist which requires indole for the efficient activation of the human receptor [43]. However, IPA is not an efficient AhR ligand [39]. Indole metabolite, skatole (3-methylindole) is an efficient ligand for AhR, but also demonstrates AhR-independent activation of p38 in intestinal epithelial cells [58]. Tryptamine mediates ionic flux in the intestines directly, via a $5-\mathrm{HT}_{4}$ receptor $\left(5-\mathrm{HT}_{4} \mathrm{R}\right)$, a $\mathrm{G}$-protein-coupled receptor (GPCR) [59]. Indoleacrylic acid (IAC) and indole 3-pyruvic acid (IPY), suppress inflammation via actions on Nrf2 and AhR, respectively [60,61]. These examples serve to illustrate the complexity of host receptor interactions within just one amino acid microbial catabolite family. Efficient metabolite-receptor interactions are usually in the micromolar range. The combinatorial effects of metabolites also have different outcomes - some together act as agonists, while other agonists, in combination, could result in antagonism of the receptor(s) [62]. Using the indole metabolite family as an example, further explored is how quantitative structure-activity relationships (QSAR), focused on PXR and AhR, may yield potential lead small molecules. It can be shown that these lead molecules are relatively safe, compared with known xenobiotic ligands for AhR and PXR, for in vivo applications. Since PXR and AhR have potent anti-inflammatory properties, 
there is a potential for developing the leads into therapeutic drugs for conditions such as inflammatory bowel disease.

\section{Quantitative Structure-Activity Relationships Between Indole(s) and Receptors, PXR, and AhR}

The correlation between a structure of xenobiotic and microbial indoles, and their capability to activate PXR and AhR receptors in vitro or in vivo is not straightforward, because there is mutual regulatory crosstalk between the AhR and the PXR. Also, numerous compounds are dual agonists for both receptors (Figure 1$)$. Whereas indole is a very low potency $\left(E_{50} \approx 1000 \mu \mathrm{M}\right)$ ligand of the PXR, its monomethylation at any position significantly increased its potency $\left(\mathrm{EC}_{50} \approx 25-75 \mu \mathrm{M}\right)$ [42]. The substitution of indole at position 3 with acetamide moiety led to medium-potency derivative, whereas the introduction of 2-hydroxyethyl, 2-aminoethyl, or acryloyl moiety, maintained feeble agonist effects. However, 3-substitutions with acetate, propionate, lactate, pyruvate, or aldehyde resulted rather in loss of even weak indole activity (manuscript submitted). The presence of indole- $\mathrm{N}$-acetamide moiety was identified as a PXR-active pharmacophore of viral polymerase inhibitors [63]. Interestingly, the combination of IPA and indole yielded much stronger intestinal anti-inflammatory effects via the PXR, as compared with the individual metabolites [43]. We designed highly potent $\left(E_{50} \approx 1 \mu \mathrm{M}\right)$ and efficacious PXR-selective agonists, by exploiting indole-based hybrid structure scaffolds. The key PXR-active pharmacophore was 1-(phenylsulfonyl)-indol-2-yl backbone. The new introduction of the indol-2-yl group led to the acquisition of the AhR activity and the AhR/ PXR-dual agonists; the removal of the phenyl sulfonyl group resulted in AhR supra-agonist compounds [64]. Eleven metabolites (all being 3-substituted indoles, formed from dietary tryptophan by human intestinal microbiota) were identified as the ligands and activators of the $\mathrm{AhR}$, substantially differing in their pharmacology parameters [39]. Highly potent ( $\left.\mathrm{EC}_{50} \approx 1 \mathrm{nM}\right)$ AhR-agonists, are based on 3-monosubstituted indoles [6-(indole-3-carbonyl)picolinonitrile and (indol-3-yl)(6-(trifluoromethyl)pyridin-2-yl)methanone] [65].

Examples of Mimicry of AhR and PXR Ligand Metabolites Derived from Microbes as Therapeutic Leads

As discussed previously, it was demonstrated that the combination of indole, together with its metabolite IPA, activates the human PXR. PXR agonism regulates intestinal inflammation via the TLR4-NFkB pathway [43]. Subsequently, based on the binding pharmacophore of indole and IPA on the PXR ligand-binding domain (LBD), a series of indole analogs arising for reaction intermediates and final products in PXR and AhR reporter assays, were tested [64]. In these studies, it was found that several indole analog synthetic intermediates (FKK1-10) were effective PXR agonists; however, only two of these, FFK2 and FKK9, had potent AhR agonist effects. In picking for selective PXR agonists, we focused on FKK5 and FKK6, based on the most favorable biophysical and biochemical activity profile. As a first example, FKK6 was further evaluated in human cell lines and intestinal organoid model systems of inflammation, and finally, in a mouse model of chemical colitis. The data suggest that FKK6 significantly abrogates indices of proinflammatory activity (IL8, lipocalin) in all model systems studied.

Furthermore, experiments in primary human intestinal biopsy tissues, obtained from patients with inflammatory bowel disease (IBD) ex vivo, are planned (personal communication, Dr Hao Li, Albert Einstein College of Medicine, Bronx, NY and Dr Efi Kokkotou, Beth Israel Deaconess Medical Center, Boston MA). A more focused approach is seen with AhR. Indole is an AhR agonist (EC $50 \sim 3 \mu \mathrm{M}$ to $1.5 \mathrm{mM}$ in luciferase reporter assays) $[66,39]$. However, at Harvard, investigators screened a chemical library of indoles and indazoles, which resulted in developing a potent hit, PY109. This small molecule is a potent $A h R$ agonist $\left(E_{50} \sim 1.2 \mathrm{nM}\right)$ and is a more potent and 
drug-like indole mimic that has demonstrated anti-inflammatory properties in mice [65]. PY109, when orally administered to mice, potently induced IL-22 and expanded tissue ILC3 and үס T cell subpopulations. The investigators found several other hits like PY108, all capable of similar actions as PY109. These design and chemical synthesis principles may be extended to the microbial synthesis of potent alkaloids that serve as AhR ligands $[67,68]$.

\section{Potential Application and Pitfalls of PXR and AhR Activating Microbial Metabolite Mimics in Human Diseases}

It is tempting to dive into classical drug development, but it is prudent to understand the effects of chronically perturbing PXR/AhR signaling homeostasis. In the case of both receptors, PXR and $A h R$, hyperstimulation of either receptor has tissue-specific effects, some that will not be beneficial for drug discovery. For example, hyperstimulation of AhR systemically can lead to anemia, a fatty liver, and developmentally, a cleft palate $[17,69]$. In this context, the aim of an AhR targeting treatment might be to restore the normal activation of the receptor, and not to overstimulate it. Similarly, systemic activation of PXR can have untoward effects with regards to unanticipated drug interactions, liver steatosis, and the potential to accelerate de novo malignancy [70].

Furthermore, as with any receptor involved in homeostasis, tachyphylaxis of response is also possible. Hence, applications of principles of clinical pharmacology then become salient in the drug development process for agents targeting these types of receptors. For example, in the case of FKK6 and PXR, it is imperative to drive PXR activation, perhaps in an intermittent manner and with local confinement. So, if we target inflammatory bowel disease, the ideal setting is distal colitis, in which FKK6 formulations could only be delivered as enemas for local effect. Alternatively, intermittent rectal bolus could be applied to avoid consistent hyperstimulation of PXR. Thus, a combination of patient and disease selection, coupled with some regional approaches for drug delivery, might be the best option to move these potent agents forward.

\section{Concluding Remarks}

This opinion article covers the feasibility of developing potential therapeutic leads using the concept of chemical biomimicry. The biomimics are of 'weak', but host targeted microbial metabolites, where the exact relationship between the ligand and its cognate therapeutic target(s) are known. It is put forward that there are some prerequisites for this to happen. First, the chemistry of deriving mimics should be simple and well defined. There should be a resolution of enantiomers, as many of these receptors have enantiomer-specific interactions. Second, the relationship between the host target and the microbial metabolite should be well defined, preferably by direct binding studies. Third, there should be a firm ability to perform classical biochemistry studies with the target. Fourth, the analog will need to be tested in terms of its affinity for binding to the receptor. If possible, the crystal structures of the ligand-bound receptor should be ascertained. Fifth, the association, in terms of cause and effect between the host target and the physiology (or pathophysiology) under therapy, should be well characterized. However, even with these prerequisites, broader questions are looming (see Outstanding Questions). For example, it is unknown as to what the true extent of weak (and promiscuous) metabolite-receptor pairings is in humans, specifically those that modify host physiology or pathophysiology. If each is mined, how many can genuinely be subjected to the prerequisites mentioned in the previous text to further drug discovery? Drug discovery is already replete with ideas that expand chemical space simply based on the limits of chemistry per se $[71,72]$. Hence, an outstanding question is whether this could help the concept develop further, or if the concept of directed metabolite mimicry enlarges the chemical space. There is much to be learned from mining microbial metabolite-host receptor interactions, and there is a ray of hope that future work will exploit these interactions favorably.
Outstanding Questions

To what extent are host receptormetabolite pairings promiscuous and weak (affinity in the micro to millimolar range)?

How many of these interactions actually influence host physiology or pathophysiology?

How many receptor-metabolite pairs that influence host physiology are actually amenable to chemical analog investigations? A major prerequisite for this would be that the ligand in question must be amenable to chemistry that is simple, and dramatically changes receptor interactions. 


\section{Acknowledgments}

Supported in part by The Peer Reviewed Medical Research Program - Investigator Initiated Research Award under Award No. W81XWH-17-1-0479; NIH grants (ES030197; CA 222469); Czech Health Research Council, grant number [NV19-05-00220] (to Z.D.).

\section{Disclaimer Statement}

S.M. and Z.D. have filed a patent application US 2019/0367475 A1 on PXR agonists and uses thereof for gut barrier dysfunction and treatment prevention.

\section{References}

1. Keah, H.H. and Hearn, M.T. (2005) A molecular recognition paradigm: promiscuity associated with the ligand-receptor interactions of the activin members of the TGF-beta superfamily. J. Mol. Recognit. 18, 385-403

2. Ekins, S. et al. (2009) Challenges predicting ligand-receptor interactions of promiscuous proteins: the nuclear recepto PXR. PLoS Comput. Biol. 5, e1000594

3. Giani Tagliabue, S. et al. (2019) Modeling the binding of diverse ligands within the Ah receptor ligand binding domain. Sci. Rep. 9, 10693

4. Sladek, F.M. (2011) What are nuclear receptor ligands? Mol. Cell. Endocrinol. 334, 3-13

5. Denison, M.S, and Nagy, S.R. (2003) Activation of the ary hydrocarbon receptor by structurally diverse exogenous and endogenous chemicals. Annu. Rev. Pharmacol. Toxicol. 43, 309-334

6. Rothhammer, V. and Quintana, F.J. (2019) The aryl hydrocarbon receptor: an environmental sensor integrating immune responses in health and disease. Nat. Rev. Immunol. 19, 184-197

7. Murray, I.A. et al. (2014) Aryl hydrocarbon receptor ligands in cancer: friend and foe. Nat. Rev. Cancer 14, 801-814

8. Metidji, A. et al. (2018) The environmental sensor AHR protects from inflammatory damage by maintaining intestinal stem cell homeostasis and barrier integrity. Immunity 49, 353-362

9. Lee, J.H. et al. (2010) A novel role for the dioxin receptor in fatty acid metabolism and hepatic steatosis. Gastroenterology 139 , 653-663

10. Peppers, J. et al. (2019) A phase 2, randomized dose-finding study of tapinarof (GSK2894512 cream) for the treatment of atopic dermatitis. J. Am. Acad. Dermatol. 80, 89-98 e3

11. Cheong, J.E. and Sun, L. (2018) Targeting the IDO1/TDO2 KYN-AhR pathway for cancer immunotherapy - challenges and opportunities. Trends Pharmacol. Sci. 39, 307-325

12. Baker, J.R. et al. (2020) The aryl hydrocarbon receptor (AhR) as a breast cancer drug target. Med. Res. Rev. 40, 972-100

13. Darakhshan, S. and Pour, A.B. (2015) Tranilast: a review of its therapeutic applications. Pharmacol. Res. 91, 15-28

14. van den Bogaard, E.H. et al. (2013) van Vlijmen-Willems IM, Hato SV, van der Valk PG, Schroder JM, Joosten I, Zeeuwen PL, Schalkwijk J. Coal tar induces AHR-dependent skin barrier repair in atopic dermatitis. J. Clin. Invest. 123, 917-927

15. Robbins, K. et al. (2019) Phase 2, randomized dose-finding study of tapinarof (GSK2894512 cream) for the treatment of plaque psoriasis. J. Am. Acad. Dermatol. 80, 714-721

16. Lamas, B. et al. (2016) CARD9 impacts colitis by altering gut microbiota metabolism of tryptophan into aryl hydrocarbon receptor ligands. Nat. Med. 22, 598-605

17. Kim, D.J. et al. (2020) Vitamin B12 and folic acid alleviate symptoms of nutritional deficiency by antagonizing aryl hydrocarbon receptor. Proc. Natl. Acad. Sci. U. S. A. 117, 15837-15845

18. Lehmann, J.M. et al. (1998) The human orphan nuclear recepto PXR is activated by compounds that regulate CYP3A4 gene expression and cause drug interactions. J. Clin. Invest. 102, 1016-1023

19. Chai, S.C. et al. (2019) Drug discovery technologies to identify and characterize modulators of the pregnane $\mathrm{X}$ receptor and the constitutive androstane receptor. Drug Discov. Today 24, 906-915

20. Chai, S.C. et al. (2020) Strategies for developing pregnane $X$ receptor antagonists: implications from metabolism to cancer. Med. Res. Rev. 40, 1061-1083
21. Forman, B.M. et al. (1998) Androstane metabolites bind to and deactivate the nuclear receptor CAR-beta. Nature 395, 612-615

22. Willson, T.M. and Kliewer, S.A. (2002) PXR, CAR and drug metabolism. Nat. Rev. Drug Discov. 1, 259-266

23. Banerjee, M. et al. (2015) Targeting xenobiotic receptors PXR and CAR in human diseases. Drug Discov. Today 20, 618-628

24. Calkin, A.C. and Tontonoz, P. (2012) Transcriptional integration of metabolism by the nuclear sterol-activated receptors LXR and FXR. Nat. Rev. Mol. Cell Biol. 13, 213-224

25. Lin, C.Y. and Gustafsson, J.A. (2015) Targeting liver $X$ receptors in cancer therapeutics. Nat. Rev. Cancer 15, 216-224

26. Hong, C. and Tontonoz, P. (2014) Liver X receptors in lipid metabolism: opportunities for drug discovery. Nat. Rev. Drug Discov. 13, 433-444

27. Kim, W. et al. (2017) Randomised clinical trial: the efficacy and safety of oltipraz, a liver $X$ receptor alpha-inhibitory dithiolethione in patients with non-alcoholic fatty liver disease. Aliment. Pharmacol. Ther. 45, 1073-1083

28. Kirchgessner, T.G. et al. (2016) Beneficial and adverse effects of an LXR agonist on human lipid and lipoprotein metabolism and circulating neutrophils. Cell Metab. 24, 223-233

29. Czarnowicki, T. et al. (2018) Effect of short-term liver $X$ receptor activation on epidermal barrier features in mild to moderate atopic dermatitis: a randomized controlled trial. Ann. Allergy Asthma Immunol. 120, 631-640 e11

30. Schaap, F.G. et al. (2014) Bile acid receptors as targets for drug development. Nat. Rev. Gastroenterol. Hepatol. 11, 55-67

31. Mudaliar, S. et al. (2013) Efficacy and safety of the farnesoid X receptor agonist obeticholic acid in patients with type 2 diabetes and nonalcoholic fatty liver disease. Gastroenterology 145 $574-582$ e1

32. Neuschwander-Tetri, B.A. et al. (2015) Farnesoid X nuclear receptor ligand obeticholic acid for non-cirrhotic, non-alcoholic steatohepatitis (FLINT): a multicentre, randomised, placebocontrolled trial. Lancet 385, 956-965

33. Kowdley, K.V. et al. (2020) A randomized, placebo-controlled, phase II study of obeticholic acid for primary sclerosing cholangitis. J. Hepatol. 73, 94-101

34. Nevens, F. et al. (2016) Group PS. A placebo-controlled trial of obeticholic acid in primary biliary cholangitis. N. Engl. J. Med. 375, 631-643

35. Peters, J.M. et al. (2012) The role of peroxisome proliferatoractivated receptors in carcinogenesis and chemoprevention. Nat. Rev. Cancer 12, 181-195

36. Hiukka, A. et al. (2010) PPARalpha: an emerging therapeutic target in diabetic microvascular damage. Nat. Rev. Endocrinol. 6, 454-463

37. Rooks, M.G. and Garrett, W.S. (2016) Gut microbiota, metabolites and host immunity. Nat. Rev. Immunol. 16, 341-352

38. Roager, H.M. and Licht, T.R. (2018) Microbial tryptophan catabolites in health and disease. Nat. Commun 9, 3294

39. Vyhlidalova, B. et al. (2020) Gut microbial catabolites of tryptophan are ligands and agonists of the aryl hydrocarbon receptor: a detailed characterization. Int. J. Mol. Sci. 21, 2614

40. Jin, U.H. et al. (2017) Short chain fatty acids enhance aryl hydrocarbon (Ah) responsiveness in mouse colonocytes and caco-2 human colon cancer cells. Sci. Rep. 7,10163

41. Pernomian, L. et al. (2020) The aryl hydrocarbon receptor (AHR) as a potential target for the control of intestinal inflammation: insights from an immune and bacteria sensor receptor. Clin. Rev. Allergy Immunol. https://doi.org/10.1007/s12016-020-08789-3 
42. Vyhlidalova, B. et al. (2020) Differential activation of human pregnane $\mathrm{X}$ receptor $\mathrm{PXR}$ by isomeric mono-methylated indoles in intestinal and hepatic in vitro models. Toxicol. Lett. 324 104-110

43. Venkatesh, M. et al. (2014) Symbiotic bacterial metabolites reg ulate gastrointestinal barrier function via the xenobiotic sensor PXR and Toll-like receptor 4. Immunity. 41, 296-310

44. Huang, K. et al. (2018) Targeting the PXR-TLR4 signaling pathway to reduce intestinal inflammation in an experimental mode of necrotizing enterocolitis. Pediatr. Res. 83, 1031-1040

45. Hudson, G.M. et al. (2017) Constitutive androstane receptor regulates the intestinal mucosal response to injury. Br. J. Pharmacol. $174,1857-1871$

46. Li, F. et al. (2013) Microbiome remodelling leads to inhibition of intestinal farnesoid $\mathrm{X}$ receptor signalling and decreased obesity. Nat. Commun. 4, 2384

47. Huang, F. et al. (2019) Theabrownin from Pu-erh tea attenuates hypercholesterolemia via modulation of gut microbiota and bile acid metabolism. Nat. Commun. 10, 4971

48. Sun, L. et al. (2018) Gut microbiota and intestinal FXR mediate the clinical benefits of metformin. Nat. Med. 24, 1919-1929

49. Quinn, R.A. et al. (2020) Global chemical effects of the microbiome include new bile-acid conjugations. Nature. 579, 123-129

50. Nepelska, M. et al. (2017) Commensal gut bacteria modulate phosphorylation-dependent PPARgamma transcriptional activity in human intestinal epithelial cells. Sci. Rep. 7, 43199

51. Byndloss, M.X. et al. (2017) Microbiota-activated PPAR-gamma signaling inhibits dysbiotic Enterobacteriaceae expansion. Science. 357, 570-575

52. Bassaganya-Riera, J. et al. (2012) Probiotic bacteria produce conjugated linoleic acid locally in the gut that targets macrophage PPAR gamma to suppress colitis. PLoS One 7, e31238

53. Borghi, M. et al. (2020) Tryptophan as a Central Hub for Host/ Microbial Symbiosis. Int J Tryptophan Res. 13, 1178646920919755

54. Agus, A. et al. (2018) Gut microbiota regulation of tryptophan metabolism in health and disease. Cell Host Microbe 23, 716-724

55. Chimerel, C. et al. (2014) Bacterial metabolite indole modulates incretin secretion from intestinal enteroendocrine $L$ cells. Cell Rep. 9, 1202-1208

56. Yang, J. et al. (2020) Biphasic chemotaxis of Escherichia coli to the microbiota metabolite indole. Proc. Natt. Acad. Sci. U. S. A. 117, 6114-6120

57. Swimm, A. et al. (2018) Indoles derived from intestinal microbiota act via type I interferon signaling to limit graft-versus-host disease. Blood 132, 2506-2519
58. Kurata, K. et al. (2019) Skatole regulates intestinal epithelial cellular functions through activating aryl hydrocarbon receptors and p38. Biochem. Biophys. Res. Commun. 510, 649-655

59. Bhattarai, Y. et al. (2018) Gut microbiota-produced tryptamine activates an epithelial G-protein-coupled receptor to increase colonic secretion. Cell Host Microbe 23, 775-785.e5

60. Wlodarska, M. et al. (2017) Indoleacrylic acid produced by commensal peptostreptococcus species suppresses inflammation. Cell Host Microbe 22, 25-37.e6

61. Aoki, R. et al. (2018) Indole-3-pyruvic acid, an aryl hydrocarbon receptor activator, suppresses experimental colitis in mice. J. Immunol. 201, 3683-3693

62. Jin, U.H. et al. (2014) Microbiome-derived tryptophan metabolites and their aryl hydrocarbon receptor-dependent agonist and antagonist activities. Mol. Pharmacol. 85, 777-788

63. Harper S. et al (2005) Potent inhibitors of subgenomic hepatitis $\mathrm{C}$ virus RNA replication through optimization of indole- $\mathrm{N}$ acetamide allosteric inhibitors of the viral NS5B polymerase. J. Med. Chem. 48, 4547-4557

64. Dvorak, Z. et al. (2020) Targeting the pregnane X receptor using microbial metabolite mimicry. EMBO Mol. Med. 12, e11621

65. Chen, J. et al. (2020) Modulation of lymphocyte-mediated tissue repair by rational design of heterocyclic aryl hydrocarbon receptor agonists. Sci. Adv. 6, eaay8230

66. Hubbard, T.D. et al. (2015) Indole and tryptophan metabolism: endogenous and dietary routes to Ah receptor activation. Drug Metab. Dispos. 43, 1522-1535

67. Mexia, N. et al. (2019) A Biomimetic, one-step transformation of simple indolic compounds to malassezia-related alkaloids with high AhR potency and efficacy. Chem. Res. Toxicol. 32, 2238-2249

68. Yu, J. et al. (2019) A tryptophan metabolite of the skin microbiota attenuates inflammation in patients with atopic dermatitis through the aryl hydrocarbon receptor. J. Allergy Clin. Immunol. 143, 2108-2119.e12

69. Turski, W.A. et al. (2020) AhR and IDO1 in pathogenesis of Covid-19 and the "Systemic AhR Activation Syndrome" translational review and therapeutic perspectives. Restor. Neurol. Neurosci. 38, 343-354

70. Biswas, A. et al. (2009) Elucidating the 'Jekyll and Hyde' nature of PXR: the case for discovering antagonists or allosteric antagonists. Pharm. Res. 26, 1807-1815

71. Naveja, J.J. and Medina-Franco, J.L. (2019) Finding constellations in chemical space through core analysis. Front. Chem. 7, 510

72. Llanos, E.J. et al. (2019) Exploration of the chemical space and its three historical regimes. Proc. Natl. Acad. Sci. U. S. A. 116 , 12660-12665 